\title{
Reflexiones de la extensión universitaria en las MSMEs desde los procesos de E-Learning
}

Reflections of the university extension in Mipymes from the E-Learning processes

Edward Fabián Escovar Alvarez

Universitaria Virtual Internacional, Bogotá, Colombia.

Correo electrónico: efescovara@uvirtual.edu.co

\section{Paula Andrea Sánchez Morales}

Universitaria Virtual Internacional, Bogotá, Colombia.

Correo electrónico: pasanchezm@uvirtual.edu.co 


\section{Resumen}

En el presente documento se discute las relaciones entre los procesos de extensión universitaria y la sociedad, puntualmente en las micro, pequeñas y medianas empresas (en adelante: Mipymes). Se parte de un enfoque histórico y epistemológico del constructo de “extensión universitaria". Posteriormente, se plantea una descripción de la situación actual de las Mipymes en Colombia y sus referentes a nivel mundial, a través de lo cual se devela la influencia y vinculación de la extensión universitaria con estos. Así mismo, se realiza una revisión de los constructos relacionados con procesos de aprendizaje virtual (E-Learning) para lograr una síntesis de la situación, desafíos y nuevas perspectivas de la extensión universitaria desde este enfoque y su impacto en las Mipymes.

Palabras clave: E-Learning, extensión universitaria, Mipymes, universidadsociedad, impacto social.

\section{Abstract}

This document discusses the relationships between the university extension processes and society, punctually in micro, small and medium enterprises (hereinafter: MSMEs). It is based on a historical and epistemological approach to the "university extension" construct. Subsequently, a description of the current situation of MSMEs in Colombia and its worldwide references is presented, through which the influence and linkage of university extension with these is revealed. Likewise, a review of the constructs related to virtual learning processes (E-Learning) is carried out to achieve a synthesis of the situation, challenges and new perspectives of the university extension from this approach and its impact on MSMEs.

Keywords: E-Learning, University extension, MSMEs, university-society, social impact.

\section{Introducción}

Referirse al concepto de extensión universitaria genera una multiplicidad de argumentos que distorsionan la construcción teórica, y su posterior aplicación como función sustantiva de las instituciones de educación superior. Así mismo, departir acerca de su reconstrucción y revitalización presenta una situación contradictoria. De un lado, la extensión no ha logrado su lugar en las agendas de gobierno, y por el otro, la preocupación por vincular esta función dentro de la sinergia universitaria es creciente (Ibragimov, Vélez-Pareja \& Tham, 2013; Tomassino \& Cano, 2016, p. 7).

Esa situación es más crítica al dilucidar la situación de diferentes sectores, como el caso de las Mipymes, que presentan una situación igualmente paradójica. Por un lado, ostentan múltiples beneficios para la sociedad, por el otro, evidencian fragilidad y carencias de competitividad en una sociedad con dinámicas de constante evolución (Confederación Colombiana de Cámaras de Comercio, 2016).

Con la intención de aportar sobre los vínculos y desafíos de la relación entre exten- 
sión universitaria y Mipymes, esta situación se aborda desde la óptica de la virtualidad, indagando sobre la pertinencia de este en la relación universidad-sociedad y poder concurrir en la delimitación de las posibilidades y dificultades de la extensión, en un entorno en el que el constructo "nuevas tecnologías" carece de alcance en una sociedad contemporánea permeada de una cultura informática (Tünnermann Bernheim, 2000, pp. 12-13).

\section{Evolución histórica de la extensión universitaria}

La extensión universitaria, en compañía de la docencia y la investigación, desarrollan la misión de las instituciones de educación superior y delimitan el rumbo de estas en la vinculación con la sociedad. La premisa fundamental va encaminada al cumplimiento del encargo social por parte de las universidades, donde cada una aporta a la construcción de una relación dialógica con la sociedad. Algunos autores datan el año 1810 como el inicio del concepto de extensión, donde surge la concepción de universidad moderna, con la creación de la universidad de Berlín en Alemania, cuyo postulado era la demostración de la producción del conocimiento (D'Andrea, Zubiría \& Sastre Vázquez, 2014; Salvador Moncada, 2007).

Lo anterior vincula la investigación y la docencia al nuevo concepto de universidad que se convirtió en fuente de inspiración (Morales, Mira \& Arias, 2010), resultando en la imposición del modelo alemán en la mayoría de las universidades del mundo a principios del siglo XX (D’Andrea, Zubiría \& Sastre Vázquez, 2014). Otros autores remontan su inicio en las políticas de extensión de 1862 en los Land Grant Colleges de los Estados Unidos, pensados para extender la educación superior a la sociedad (Ángeles (1992) citado en: D’Andrea, Zubiría \& Sastre Vázquez, 2014).

Con la revolución industrial, se gestan programas formativos para adultos, creándose en 1842 el primer colegio de clase obrera en Sheffield. Posteriormente, en 1867 se establece el primer programa de extensión en Cambridge, donde se empezó a reconocer las responsabilidades de la universidad hacia la comunidad (D’Andrea, Zubiría \& Sastre Vázquez, 2014; Giménez Martínez, 2000; Ortiz-Riaga \& Morales-Rubiano, 2011). En 1869, se crean cursos y conferencias en varias universidades de Alemania, que le dan continuidad a la política de extensión que se viene desarrollando. Para 1900 se crea la facultad de extensión universitaria en la Universidad de Chicago, y en 1902 se inauguran cursos de extensión universitaria en la universidad de Valencia (D'Andrea Zubiría \& Sastre Vázquez, 2014).

En América Latina, al igual que en el resto del mundo, la función de extensión se ha comportado de forma heterogénea, tanto en su concepción desde la institucionalidad, como en su enfoque estratégico (2011, p. 354). El principal avance se ha dado desde las manifestaciones culturales, sin prosperar en la creación de un espacio de acción y vinculación con la investigación y la docencia. Uno de los primeros adelantos en Latinoamérica en extensión se dio en 1907 en la Universidad de la Plata, donde se desarrollaron conferencias de extensión universitaria, y para Colombia en 1909, con el discurso de Rafael Uribe Uribe donde se propone el ideal de universidad científica, moderna, experimental, actual y evolutiva (León, 2015). 
Sin embargo, fue con la reforma de Córdoba en 1918, que se comenzó a cuestionar el carácter social de la universidad, allí se buscaron logros de carácter político-social, inclusión de la clase media, autonomía universitaria, fortalecimiento de la función social de la universidad (extensión universitaria), entre otros aspectos. Con lo cual se estructura la denominada "misión social de la universidad" (Angeles, 1992; Carlevaro, 2008; González González \& González Fernández-Larrea, 2003; León, 2015; Morales, Mira \& Arias, 2010; Salvador Moncada, 2007; Tünnermann Bernheim, 2000). Posteriormente, se despliega en Latinoamérica una serie de actividades encaminadas a fortalecer esta nueva conducta universitaria, ahora con un enfoque más crítico y propositivo.

En 1921 se celebra el congreso internacional de estudiantes en México, donde se trabaja la extensión como obligación del estudiantado. En 1923 se incorpora la idea de extensión dentro de la legislación de la Universidad Católica de Chile. En 1924 se establece el departamento de extensión en la Universidad de México, y en el año 1929 se adhiere dentro de la ley orgánica de la Universidad Nacional de México, al igual que en Chile es aprobado el estatuto orgánico de enseñanza universitaria, donde entre otros aspectos, es definido el papel de la universidad en el campo de la extensión. Años más tarde, en la Universidad de Chile se gesta la reforma de 1931 donde uno de sus componentes era la difusión de cultura (León, 2015).

Sobre el año 1944 la Universidad Nacional de Colombia fortalece las relaciones entre la universidad y la sociedad. En 1945 se crea la Ley Orgánica de la UNAM, donde se sentaron las bases para la política de extensión de esta universidad. Así mismo, en 1949 la Universi- dad de San Carlos de Guatemala desarrolló el Primer Congreso de Universidades Latinoamericanas, donde se enunció que la función de la extensión debía atender la ciencia, la literatura y el arte, y donde se ratificó la función social de la universidad y su expresión a través de la extensión (Angeles, 1992, p. 4; León, 2015).

Uno de los esfuerzos más influyentes en la conceptualización de la extensión universitaria fue la Primera conferencia Latinoamericana de Extensión Universitaria y Difusión Cultural en 1957, donde se reconoce como una agrupación de acciones con vocación de proyectar dinámica y vincular a la sociedad (Angeles, 1992, p. 4; Fresán Orozco, 2004, p. 47). Posteriormente hacia el año 1958 se crea el departamento de extensión universitaria en la Universidad de Buenos Aires (León, 2015). La segunda Conferencia Latinoamericana de Extensión Universitaria y Difusión Cultural desarrollada en México en 1972 cuestiona el carácter asistencialista de la extensión y su falta de participación en la sociedad, y propone un enfoque liberador con sentido de concientización de los individuos sobre su realidad, para lograr transformarla. Se busca adoptar un nuevo enfoque sin jerarquía entre los individuos, y donde los mensajes se manejen de forma dialógica (Angeles, 1992, p. 5).

En la década de los años 80 en Colombia, se reconoce la extensión formalmente a través del Decreto 80 (Ministerio de Educación Nacional, 1980), dando inicio al desarrollo de esta función en el país (León, 2015; Morales, Mira \& Arias, 2010). En esta misma década, los enfoques de acción participativa cobran mayor relevancia en conjunto con el continuo trabajo por un mayor relacionamiento con el 
entorno social (García, 2008). Para los noventa, la extensión universitaria empezó a adquirir un impulso distinto, adhiriendo a su concepción la articulación de la universidad con los sectores productivos, ejemplo de esto es la Ley 30 de 1992 promulgada en Colombia, que en el artículo 120 define la extensión y da paso para que posteriormente la pertinencia social empezará a ser equiparada con la satisfacción de las necesidades del mercado (León, 2015; Morales, Mira \& Arias, 2010).

En 1992 en la Universidad de la República en Uruguay, se gesta un programa denominado Ápex-Cerro cuyo principal interés es vincular el aprendizaje con la extensión (2008, p. 26). En esta misma década continuó la preocupación de una universidad con función social e inclusivo, donde se creara una cultura de extensión, no como añadidura, sino como razón de ser (García, 2008). Esta preocupación ha continuado y ha generado una búsqueda de mecanismos de integración universidades-sociedad en Latinoamérica, que ha permitido la vinculación de la extensión dentro de los estamentos universitarios y se ha posibilitado la creación de espacios de reflexión como los Congresos Latinoamericanos de Extensión Universitaria o la creación de la Unión Latinoamericana de Extensión Universitaria (González González \& González Fernández-Larrea, 2003).

\section{La extensión universitaria como función sustantiva}

La tercera función sustantiva conocida principalmente como extensión universitaria, presenta una multiplicidad de denominaciones que dependen de su historicidad y su contexto de enunciación (Buenfil citado en Tomassino \& Cano, 2016), encontrándose referencias a esta como "proyección social", "proyección universitaria", "divulgación cultural", "misión social", "difusión cultural", "interacción social" entre otros (D'Andrea, Zubiría \& Sastre Vázquez, 2014; Ruiz, 2016). La polisemia constitutiva, adicional a las variadas tareas que le han asignado a esta función y la autodefinición que se da en cada universidad, en su visión de relacionamiento con la sociedad, ha distorsionado la conceptualización y profundización del concepto (Fresán Orozco, 2004; Gezmet, 2013).

Vega Mederos (2002), González González \& González Fernández-Larrea (2003) y García (2008) presentan algunas limitaciones u obstáculos que se han presentado en el desarrollo de la extensión, de las cuales se destacan: diversidad de denominaciones, falta de presupuesto e infraestructura, ausencia de una cultura extensionista (marginación frente a las otras funciones sustantivas), incongruencia entre el discurso y la acción de las autoridades universitarias, visión asistencialista y complementaria, mayor focalización en relación universidad-industria que universidad-sociedad (carácter neoliberal) y no integración de las tres funciones sustantivas. Dando evidencia de los retos por adelantar para lograr una función extensionista con un enfoque social.

Para superar estas falencias, se han venido consolidando enfoques que permiten comprender el desarrollo de la extensión universitaria, y los aportes que cada uno ha otorgado al encargo social de las universidades. Se pueden encontrar cinco modelos que han delineado la extensión universitaria (García, 2008; Freire, 1979; Gezmet, 2013; González González \& González Fernández-Larrea, 2003; Ortiz-Riaga \& 
Morales-Rubiano, 2011; Ruiz Lugo, 1992; Serna Alcántara, 2004; Serna Alcántara, 2007; Tommasino, González Márquez, Guedes, \& Prieto, 2006; Vega Mederos, 2002):

- Altruista: en este enfoque encajan las primeras acciones de las universidades, cuya principal característica era el desinterés-voluntad de los miembros de estas.

- Divulgativo: que buscaba aproximar la sociedad a todos los avances culturales y técnicos generados al interior de la universidad. A este enfoque se le asocian fuertes críticas sobre la ausencia de visión sobre las verdaderas necesidades de la sociedad.

- Concientizador: también denominada extensión crítica, es una corriente heredada de la pedagogía freiriana con postulados sobre la creación de conciencia, capacidad de análisis crítico, acción transformadora, y la comunicación dialógica y liberadora, logrando una práctica extensionista que permita contextualizar el problema para comprenderlo y transformarlo conjuntamente. Lo anterior garantizará que las necesidades sociales demandadas por los actores puedan ser abordadas de forma idónea, dado que, en la mayoría de los casos, las soluciones y explicaciones no estarán contextualizadas o regladas en el currículo (Tommasino, Santos, \& Stevenazzi, 2010).

La principal crítica que germina de este enfoque es la unidireccionalidad que tiene la extensión universitaria: en un lado están los que dan, y en el otro quienes reciben, sin lograr esa simbiosis de aprendizajes.

- Vinculatorio empresarial: que parte de equiparar las necesidades sociales con las necesidades corporativas, direccionando el sistema educativo a cubrir esas nuevas necesidades, generando la exclusión de aquellos que no pueden cubrir los costos de esos servicios. El resultado es una universidad cómplice de un sistema deshumanizado y salvaje donde solo se admiten intereses de orden económico. Este enfoque extensionista predomina en las universidades de Europa Occidental y los Estados Unidos donde persisten en el desarrollo industrial, así como a la concepción de la prestación de sus servicios (González González \& González Fernández-Larrea, 2003).

- Desarrollo integral: se define bajo la óptica de una universidad democrática, crítica y creativa, cimentada en el concepto de la democratización del saber, que al igual que el modelo concientizador se piensa sobre un diálogo multidireccional con los diferentes actores involucrados, bajo la óptica de una interacción social que conlleve a una igualdad de las partes y que ambas logren un desarrollo y se enriquezcan.

Los modelos "concientizador" y "desarrollo integral" plantean algunos desafíos que las universidades deben atender para poder hablar de un impacto real en la sociedad (Malagón Plata, 2012), los cuales se pueden agrupar de la siguiente forma: 
- Abordaje interdisciplinario y multiprofesional, dado que esa relación dialógica y crítica que se plantea entre los actores involucrados propone un vínculo dialéctico de saberes académicos y populares.

- Reto a la apertura dado que esa realidad contextualizada postula preguntas que el acto educativo no ha dimensionado (Tomassino \& Cano, 2016; Tommasino, Santos, \& Stevenazzi, 2010), lo que requiere una vinculación de las tres funciones sustantivas que permita una articulación de esfuerzos que dé respuesta a las verdaderas necesidades sociales (Morales, Mira \& Arias, 2010; Tomassino \& Cano, 2016, pp. 16-17)

- Pensar la relación universidad-sociedad como un proceso de encuentro y comunicación sin que se convierta en una invasión cultural y una imposición de un sistema de valores, y donde no se reconoce la particularidad (Carlevaro, 2008; Tomassino \& Cano, 2016; Tommasino, González Márquez, Guedes, \& Prieto, 2010). Esta relación debe romper con el aislamiento de la universidad, debe generar un compromiso que se transforme en acción y participación por parte de los involucrados (Navarro de Gottifredi, 2002).

Un elemento central de los modelos "concientizador y "desarrollo integral" es el concepto de concientización al que Freire (1972) hace referencia como un proceso de conciencia de las realidades sociales y de sus posibilidades emancipatorias. Este concepto requiere un actuar que apunte a una relación de acción-reflexión (Ruiz,
2007, p. 6; Serna Alcántara, 2004, p. 89; Tommasino, González Márquez, Guedes, \& Prieto, 2006, p. 130). El pensar en la extensión requiere tener una concepción amplia de las bondades de interconectarla con la docencia y la investigación. Bajo esta perspectiva, adquiere una función estratégica en cuanto a la capacidad de articulación y la posibilidad de una formación integral de los estudiantes y de los demás miembros de la comunidad universitaria (2004, p. 47), es así como realmente se lograría consolidar un desarrollo en las universidades (2002). Para que la extensión adquiera un carácter estratégico es necesario que se dimensionen los objetivos que las universidades deben replantearse en su quehacer en el campo de la extensión (Angeles, 1992; Serna Alcántara, 2007; Vega Mederos, 2002):

- Articulación de la docencia y la investigación con las demandas intra y extrauniversitaria, que permita la vinculación constante entre teoría y práctica.

- Democratizar el conocimiento académico y la participación efectiva de la sociedad, enfocada en la población marginada y vulnerable.

- Incentivar la practica académica en pro de contribuir en el desarrollo de la conciencia social y política.

- Participar críticamente de las propuestas que apunten al desarrollo regional, económico, social, ambiental y cultural.

- Estimular la cooperación interinstitucional y la producción de material de divulgación adecuado a las diferentes áreas del conocimiento. 


\section{Micros, pequeñas y medianas empresas}

El artículo segundo de la Ley 590 del 2000, denominada la ley de desarrollo de las micros, pequeñas y medianas empresas, modificada por la Ley 1450 de 2011, categoriza las empresas en Colombia y realiza una primera aproximación al concepto de empresa indicando que (2000): "se entiende por empresa, toda unidad de explotación económica, realizada por persona natural o jurídica, en actividades agropecuarias, industriales, comerciales o de servicios, en el área rural o urbana". Así mismo, se indica que para Colombia la categorización se dará entre micro, pequeña, mediana y gran empresa, teniendo en cuenta factores como el número de trabajadores totales, el valor de las ventas brutas anuales y el valor de los activos totales (Congreso de la República, 2000). Esta clasificación presenta desviaciones, frente a las variables utilizadas en otros países, lo que dificultad el análisis comparativo (Vera \& Mora, 2011; Zevallos, 2003) y limita los esfuerzos por generar políticas macro por parte de entidades multilaterales.

La caracterización empresarial en Colombia, la cual fue modificada por la Ley 905 del 2.004, se presenta de acuerdo con la siguiente tabla (2000):

Tabla 1. Criterios de clasificación empresarial en Colombia.

\begin{tabular}{|l|l|l|l|l|}
\hline & Microempresa & Pequeña empresa & Mediana empresa & Gran empresa \\
\hline $\begin{array}{l}\text { Número de emplea- } \\
\text { dos }\end{array}$ & $1-10$ Trabajadores & 11-50 Trabajadores & $\begin{array}{l}51-200 \text { Trabaja- } \\
\text { dores }\end{array}$ & $>$ 200 Trabajadores \\
\hline Activos totales & $\begin{array}{l}\text { Hasta 500 SMMLV* } \\
\text { (Excluida la vivienda) }\end{array}$ & $\begin{array}{l}\text { Superior a } 500 \text { y has- } \\
\text { ta 5.000 SMMLV }\end{array}$ & $\begin{array}{l}\text { Superior a 5.000 y } \\
\text { hasta 15.000 SMMLV }\end{array}$ & $\begin{array}{l}\text { Mayor o igual } 15.000 \\
\text { SMMLV }\end{array}$ \\
\hline *SMMLV = Salario Mínimo Mensual Legal Vigente \\
\hline
\end{tabular}

La anterior clasificación evidencia una necesaria focalización de esfuerzos, por la indiscutible segregación de necesidades que acogen a cada uno de los grupos. Muestra de lo anterior, son las tasas de supervivencia que afecta cada uno de los grupos de empresas, las grandes empresas mantienen una tasa de supervivencia promedio del $71,4 \%$, y a medida que el tamaño va bajando, el porcentaje promedio de supervivencia también lo hace, pasando por un $68 \%$ para las medianas empresas, $60 \%$ para las pequeñas y tan solo el $29,1 \%$ para las microempresas (2016, p. 6). La necesidad de políticas públicas es inminente al comparar el país frente a referentes regionales y mundiales, donde se evidencia índices de supervivencia empresarial a 5 años inferiores a los presentados por países de la OCDE, grupo al que Colombia se integró en el año 2018, como es el caso de Chile o Argentina con índices del $49,4 \%$ y $49,1 \%$ respectivamente, o referentes como Bélgica $(82,2 \%)$ y Finlandia $(63,5 \%)$ que denotan un arduo trabajo por hacer en materia de fortalecimiento empresarial (Confede- 
ración Colombiana de Cámaras de Comercio, 2017, p. 9).

Adicionalmente, al revisar la composición empresarial del país por antigüedad, se encuentra como cerca del $32,4 \%$ del engranaje empresarial está conformado por Startups que no tienen más de 2 años, el 24\% está entre 2 y 5 años, el 20,6\% entre 5 y 10 años, y el 23\% de empresas con una antigüedad superior al 10 años (Confederación Colombiana de Cámaras de Comercio, 2018, pp. 4-5). Lo anterior, enlazado con la tasa de supervivencia empresarial en el país, hace un llamado a la necesidad de contextualizar los requerimientos que las micros, pequeñas y medianas empresas demandan. Entre el 2013 y el 2017, se crearon 1.075.908 empresas y se disolvieron 1.011.613, resultando que para final del 2017 en el país se encontraban registradas y funcionando un total 1.378.194 empresas, lo que representa una Tasa de Entrada Bruta (TEB) promedio del 19,2\% y una Tasa de Salida Bruta (TSB) promedio del $18,1 \%$, lo que significa que por cada 100 empresas funcionando, se formalizan $19 \mathrm{y}$ se disuelven 18 en promedio (Confederación Colombiana de Cámaras de Comercio, 2018, p. 15).

Este comportamiento empresarial está fuertemente influenciado por las microempresas que durante el periodo 2014-2017 presentaron una entrada promedio del $21,1 \%$, las cuales, como se mencionó anteriormente son las que sufren de la más alta mortalidad empresarial (Confederación Colombiana de Cámaras de Comercio, 2018, p. 16). Algunos de los factores que influyen sobre la supervivencia de las empresas, y sobre los cuales se debe trabajar de forma mancomunada son: (I) los factores específicos de las compañías, que incluye el tama- ño, el sector económico, estructura financiera inicial, la orientación al mercado externo, entre otras; (II) factores sectoriales o industriales como el crecimiento en la producción o ventas de la industria, la estructura de competencia y los flujos de entrada de nuevas empresas al mercado; y (III) factores geográficos como el grado de desarrollo de la región donde opera la firma, si pertenece a algún clúster regional, entre otros (Confederación Colombiana de Cámaras de Comercio, 2017).

\section{Mipymes y extensión universitaria}

De forma generalizada, las Mipymes representan la mayor parte del tejido empresarial, y sus aportes a nivel económico y social, las sitúa como un foco de interés para los gobiernos y las entidades multilaterales. Según cifras de la Organización para la Cooperación y el Desarrollo Económico (En adelante: OECD) para el año 2013, la participación de estas empresas era del 99,7\%, el aporte a la generación de empleo era cercana al $60 \%$, y contribuían aproximadamente en una quinta a una sexta parte de la producción, (2017). Para el caso de Colombia, en el 2016 la participación de las Mipymes en el engranaje empresarial era cercano al 99,6\%, el aporte a la generación de empleo es de cerca del $80,8 \%$ y del $45 \%$ de la producción del país (MinCIT, 2016).

Esta contribución por parte de las MiPymes a nivel social y económico ha agudizado la necesidad de comprender los factores que inciden de alguna forma en la productividad y la supervivencia de estas. Dentro de los factores específicos de las empresas a la hora de 
hablar de niveles de supervivencia (Confederación Colombiana de Cámaras de Comercio, 2017), se pueden incluir factores como los niveles de educación y especialización de los grupos de trabajo o la experiencia en el sector al que pertenece la compañía, que tienen un vínculo directo con la elección de la estrategia corporativa, la cual puede inferir en la muerte empresarial que se puede dar al ejecutar decisiones caracterizadas por (Capera \& Huertas, 2018, p. 55; Tascón \& Castaño, 2013): (I) prácticas indebidas en el manejo financiero de la empresa, (II) falta de calidad de los productos y servicios, o (III) crecimiento excesivo del endeudamiento, (IV) mala gestión y deficiencias en el sistema contable, (V) crecimiento no sostenible, entre otros.

Lo anterior precisa la imperante necesidad de vincular el conocimiento que la docencia y la investigación desarrollan al interior de las universidades (Angeles, 1992; Serna Alcántara, 2007; Vega Mederos, 2002), no pensando en la postura tradicional de una transferencia descontextualizada y con ímpetu de invasión, sino con el ánimo de co-creación, de un desarrollo tanto teórico como práctico que aporte en la solución de las verdaderas necesidades de este grupo social, y que invite a una participación efectiva de las Mipymes, que como se puede concluir, tienen unos resultados que evidencian fragilidad y desenfoque organizacional. Esta vinculación no debe pensarse de forma convencional, dado que los actuales resultados de las Mipymes, a nivel mundial, y en el caso de Colombia, comparados con los esfuerzos que entidades como el Banco de la República, Cámara de Comercio, Acopi, entre otras han hecho no han dado la tranquilidad y satisfacción que se esperaba.

\section{Aportes desde la virtualidad}

Uno de los retos actuales a los cuales no son ajenas las universidades, y mucho menos las empresas, es el proceso de virtualidad que se está convirtiendo en un pilar fundamental en diferentes aspectos de la sociedad, dado que, a través de este, la espacialidad y temporalidad que limitaban muchos de los escenarios de interrelación comienzan a verse traspasados por nuevas dinámicas, en donde las tecnologías de la información y la comunicación (en adelante: TIC) configuran los procesos de intercambio de información, permiten una comunicación más efectiva y también adquieren relevancia en la intervención y mejora de los procesos formativos, dando paso a nuevos escenarios educativos, unos entornos dinámicos y en constante evolución que garantizan la construcción de ideas, la colaboración y la interpretación en grupos de trabajo (Coll, 2004; Wenger, 2001).

Dentro de este gran espectro de posibilidades, para el campo de la formación y de la extensión se encuentran herramientas como las plataformas de E-learning o Learning Management System (LMS), que consisten en unos espacios de aprendizaje virtuales que garanticen la interrelación entre tutores-alumnos a través de diferentes herramientas como los foros, chats, evaluaciones. Con lo anterior, se logra una ruptura de diferentes limitantes de la intervención presencial, y se garantiza que en el caso de la formación para empresas se logre una transferencia de conocimientos acordes a las necesidades de sus colaboradores logrando así alcanzar altos niveles de desempeño en sus funciones (Batalla-Busquets \& Pache- 
co-Bernal, 2013; Rendón Siempira, 2015; Rodríguez-Molina, 2011). Desde el punto de vista del empleado es importante que esa capacitación o formación se desarrolle en concordancia con las expectativas del trabajador, con sus creencias y competencias tanto profesionales como personales (Rabak \& Cleveland-Innes, 2006).

Sin embargo, el hablar de E-learning o educación virtual en las Mipymes debe expresarse desde la integralidad de la compañía, dado que su funcionalidad no solo favorece al personal operativo, también permite fortalecer el área gerencial en aspectos como la comunicación, planificación, gestión, entre otras (Thomas \& Sireno, 1980), adicional a los múltiples beneficios que se pueden generar en el campo de la investigación, donde las prácticas profesionales, a través de la extensión, se transformen en investigación colaborativa (Perkmann \& Walsh, 2007) y crear redes de apoyo a través de plataformas E-learning que permitan la integración del conocimiento en la vida laboral. Es a través de esa vinculación entre virtualidad y las funciones sustantivas que se puede facilitar los procesos de transferencia e interacción entre las Mipymes y la universidad, logrando una construcción de conocimiento contextualizado y una solución a las necesidades reales de estos individuos (Ankrah, Burgess, Grimshaw, \& Shaw, 2013).

\section{Conclusiones}

Como se pudo evidenciar en el presente documento, la extensión universitaria como función sustantiva se debe convertir en el vínculo y la herramienta que garantice los procesos de reflexión por parte de la universidad con la realidad de la sociedad, permitiendo que esta reflexión se transforme en dinámicas de transformación y cooperación entre todos los involucrados. Así mismo, esta función sustantiva se debe integrar junto a la investigación y la extensión, para focalizar la razón de ser de las universidades hacia la promulgación del cambio social. Un cambio en el que las universidades son las máximas protagonistas de promover y mantener el acervo cultural de la sociedad, la transformación social y la emancipación de los grupos más vulnerables.

La universidad y su interacción con la sociedad se debe mantener separados de los intereses económicos, el conocimiento no se puede convertir en mercancía bajo los lineamientos del neoliberalismo, a pesar de que la carrera hacia una universidad con un carácter empresarial parece inalterable, es importante que se siga construyendo y validando el constructo de "extensión" bajo los postulados de una universidad incluyente, social y con capacidad de transformación. Esta tarea de construir sobre ideas ya formadas no ha finalizado, la extensión como ideal de cambio no es un concepto acabado, en cambio, debe estar en permanente construcción y resignificación dado que las necesidades sociales que lo fundamentan no terminan de germinar y renovarse.

Las Mipymes enfrentan grandes retos para su sostenibilidad, y la formación bajo una modalidad virtual otorga herramientas que les permiten adquirir conocimientos los cuales pueden aplicar en sus heterogéneas necesidades, ya que tanto trabajadores como propietarios pueden capacitarse en el sitio de trabajo y con esto disminuir el riesgo del fracaso de este tipo de empresas. Al ser la educación virtual más accesible a todos los usuarios, sin im- 
portar el lugar o condición social, se logra una mayor democratización de la educación en la que actores como las Mipymes que no disponen un gran presupuesto para invertir en educación, puedan capacitarse y obtener mejores resultados, y al mismo tiempo se conviertan en generadores de conocimiento y participes en los procesos de co-creación de soluciones que requiere el país.

Es importante seguir trabajando en la búsqueda de soluciones contextualizadas para cada uno de los individuos que conforman nuestra sociedad, en el caso de las Mipymes es pertinente seguir revisando como a través de la relación investigación-extensión-docencia, se logran disminuir las problemáticas que atraviesan actualmente, y como la virtualidad debe focalizar los esfuerzos para que en la solución de estas problemáticas no se presenten barreras de espacialidad o presupuestales. Así mismo, se debe continuar con las investigaciones que realcen el aspecto social en la relación universidad-sociedad que mitigue los efectos nocivos que la visión economicista puede generar sobre el que hacer universitario.

\section{Referencias}

Angeles G., O. (1992). Consideraciones en torno al concepto de extensión de la cultura y de los servicios. Revista de la Educación Superior, 21(81), 1-9. Recuperado de: http://publicaciones.anuies.mx/pdfs/revista/Revista81_ S1A3ES.pdf

Ankrah, S. N., Burgess, T. F., Grimshaw, P. \& Shaw, N. E. (2013). Asking both university and industry actors about their engagement in knowledge transfer: What single-group studies of motives omit. Technovation, 33(2-3), 50-65. DOI: https://doi.org/10.1016/j.technovation.2012.11.001

Batalla-Busquets, J. M. \& Pacheco-Bernal, C. (2013). On-the-job E-learning: Workers' Attitudes and Perceptions. The International Review of Research in Open and Distributed Learning, 14(1), 40-64. DOI: https://doi.org/10.19173/ irrodl.v14i1.1304

Capera, I. \& Huertas A., L. E. (2018). ¿Por qué mueren las empresas? Un estudio de la interrupción de la perdurabilidad empresarial en una empresa de tecnología. Tesis de grado, Universidad del Rosario: Bogotá. Recuperado de: http://repository.urosario.edu.co/handle/10336/14251

Carlevaro, P. V. (2008). Universidad y sociedad proyección y vínculos. Reencuentro, 52, 1937. Recuperado de: http://www.documentos. una.ac.cr/bitstream/handle/unadocs/8129/ Universidad $\% 20 y \% 20$ sociedad-proyecci\%C3\%B3n\%20y\%20v\%C3\%ADnculos_Pablo\%20 Carlevaro.pdf?sequence $=16 \&$ isAllowed $=y$

García, C. (2008). El compromiso social de las universidades. Cuadernos del Cendes, 25(67), 129-134. Recuperado de: http://www.redalyc.org/articulo.oa?id=40306707

Coll, C. (2004). Psicología de la educación y prácticas educativas mediadas por las tecnologías de la información y la comunicación: una mirada constructivista. Sinéctica, $25,1-24$

Confederación Colombiana de Cámaras de Comercio. (2016). Nacimiento y Supervivencia 
de las Empresas en Colombia. Recuperado de: http://www.confecamaras.org.co/cooperacion-y-competitividad/analisis-economico

Confederación Colombiana de Cámaras de Comercio. (2017). Determinantes del crecimiento acelerado de las empresas en Colombia, 24. Recuperado de: http:// www.confecamaras.org.co/phocadownload/2018/Cuadernos_An\%C3\%A1lisis_ Econ\%C3\%B3mico/Cuaderno_de_Determinantes_de_la_productividad/Cartilla\%20 Determinantes\%20Agosto\%2024-1\%20OK. pdf

Confederación Colombiana de Cámaras de Comercio. (2018). Nuevos hallazgos de la supervivencia y crecimiento de las empresas en Colombia, 32. Recuperado de: http:// www.confecamaras.org.co/phocadownload/2018/Cuadernos_Análisis_Económico/Cuaderno_demografia_empresarial/ Cartilla17.pdf

Congreso de la República. (2000). Ley 590 de 2000. Reglamentada por el Decreto Nacional 2473 de 2010, Reglamentada por el Decreto Nacional 957 de 2019. Por la cual se dictan disposiciones para promover el desarrollo de las micro, pequeñas y medianas empresa. Régimen Legal de Bogotá. Recuperado de: https://www.alcaldiabogota. gov.co/sisjur/normas/Norma1.jsp?i=12672

D’Andrea, R. E., Zubiría, A. \& Sastre Vázquez, P. (2014). Reseña histórica de la extensión universitaria. Secretaría de la Universidad Nacional del Centro de la provincia de Buenos Aires, 33, 1-12. Recuperado de: http://extension.unicen.edu.ar/jem/completas/188.pdf
Freire, P. (1979). La pedagogía del oprimido. México: Siglo XXI.

Fresán Orozco, M. (2004). La extensión universitaria y la universidad pública. Reencuentro, 39, 47-54. Recuperado de: http:// redalyc.uaemex.mx/src/inicio/ArtPdfRed. jsp?iCve $=34003906$

Gezmet, S. (2013). La vinculación universidad-sociedad. Modelos de extensión y características de las interacciones. Compendio Bibliográfico, 24-30. Recuperado de: https:// www.unc.edu.ar/sites/default/files/MODELOS\%20DE\%20EXTENSI\%C3\%93N.pdf

Giménez Martínez, J. (2000). La extensión universitaria en España. $V$ Congreso Iberoamericano de Extensión, México. OEI. Recuperado de: https://www.oei.es/historico/ superiordoc.htm

González, G. R. \& Fernández-Larrea, M. (2002). La gestión de la extensión universitaria: una aproximación desde la perspectiva cubana. Revista Cubana de Educación Superior, 22(2), 55-68.

González González, G. R., \& González Fernández-Larrea, M. (2003). Extensión universitaria: principales tendencias en su evolución y desarrollo. Revista Cubana de Educación Superior. Recuperado de: https://link.galegroup. com/apps/doc/A146790868/AONE?u=googlescholar\&sid=AONE\&xid=e6236d6b

Ibragimov, R., Vélez-Pareja, I. \& Tham, J. (2013). VAIC: New Financial Performance Metric and Valuation Tool. Cuadernos Latinoamericanos de Administración. IX(16), 9-20. 
León, A. L. (2015). La extensión universitaria en América Latina desde sus orígenes a la actualidad. XIII Congreso Latinoamericano de Extensión Universitaria.

Malagón Plata, L. A. (2012). La vinculación universidad-sociedad desde una perspectiva social. Educación y Educadores, 9(2), 79-93. Recuperado de: https://educacionyeducadores.unisabana.edu.co/index.php/eye/article/view/664/749

Ministerio de Educación Nacional. (1980). Decreto 80 . Por el cual se organiza el sistema de educación postsecundaria. Bogotá D.C. Recuperado de: https://www.mineducacion.gov.co/1759/w3-article-102556.html? noredirect $=1$

Morales, M. E., Mira, G. E. \& Arias, M. A. (2010). Enfoques y retos de la función de extensión universitaria, como mecanismo de integración: Universidad, Empresa, Estado. II Congreso Internacional de Gestión Tecnológica e Innovación, 9.

Navarro de Gottifredi, A. M. (2002). Una forma de profundizar la relación universidad-sociedad, Cuadernos de la Facultad de Humanidades y Ciencias Sociales, 15, 31-41. Recuperado de: https://www.redalyc.org/ articulo.oa?id=18501504

OECD. (2017). Small, Medium, Strong. Trends in SME Performance and Business Conditions. Recuperado de: https://doi.org/http://dx. doi.org/10.1787/9789264275683-en

Ortiz-Riaga, M. C. \& Morales-Rubiano, M. (2011). La extensión universitaria en América Latina: concepciones y tendencias.
Educación y Educadores, 14(2), 349-366. DOI: 10.5294/edu.2011.14.2.6

Perkmann, M. \& Walsh, K. (2007). University-industry relationships and open innovation: Towards a research agenda. International Journal of Management Reviews, 9(4), 259-280. DOI: https://doi.org/10.1111/ j.1468-2370.2007.00225.x

Rabak, L. \& Cleveland-Innes, M. (2006). Acceptance and Resistance to Corporate E-Learning: A Case From the Retail Sector. Journal of Distance Education, 21(2), 115-134. Recuperado de: https://files.eric.ed.gov/ fulltext/EJ807806.pdf

Rendón Siempira, A. V. (2015). El capital humano y los programas e-learning en la formación de trabajadores. Bogotá: Universidad Pedagógica Nacional.

Rodríguez-Molina, G. (2011). Funciones y rasgos del liderazgo pedagógico en los centros de enseñanza. Educación y Educadores, 14(2), 253-267. Recuperado de: https:// www.redalyc.org/pdf/834/83421404003. pdf

Ruiz, B. M. (2007). La pedagogía crítica y Paulo Freire: aportaciones conceptuales. Revista de Ciencias de la Educación, 212, 397-408.

Ruiz Lugo, L. (1992). La extensión de la cultura y los servicios en las universidades públicas del país. Revista de la Educación Superior, 20. Recuperado de: http://publicaciones.anuies.mx/pdfs/revista/Revista81_S1A1ES.pdf 
Ruiz, M. R. (2016). Acerca de la II Conferencia Latinoamericana de difusión cultural y extensión universitaria (1972). Un debate vigente, 6, 72-82.

Salvador Moncada, J. (2007). La universidad: un acercamiento histórico-filosófico. Theoria, 16(2), 33-46. Recuperado de: http://www. ubiobio.cl/miweb/webfile/media/194/v/ v16-2/a3.pdf

Serna Alcántara, G. (2004). Modelos de extensión universitaria en México. Revista de la Educación Superior, XXXIII(3-131),26-32. Recuperado de: http://publicaciones.anuies. mx/acervo/revsup/131/02.html

Serna Alcántara, G. A. (2007). Misión social y modelos de extensión universitaria: del entusiasmo al desdén. Revista Iberoamericana de Educación, 43(3), 22-31. Recuperado de: http://beu.extension.unicen.edu.ar/ xmlui/bitstream/handle/123456789/114/ Misión social y modelos de extensión universitaria del entusiasmo al desdén.pd$\mathrm{f}$ ?sequence $=1$

Tascón Fernández, M. \& Castaño Gutiérrez, F. (2013). Variables y modelos para la identificación y predicción del fracaso empresarial: revisión de la investigación empírica Reciente. Revista de Contabilidad, 15(1), 7-58. DOI: https://doi.org/10.1016/s11384891(12)70037-7

Thomas, J, \& Sireno, P. (1980). Training Needs of Managers of Small Manufacturing Firms. American Journal of Small Business, 38-44. DOI: https://doi.org/https://doi. org/10.1177/104225878000400307
Tomassino, H., \& Cano, A. (2016). Modelos de extensión universitaria en las universidades latinoamericanas en el siglo XXI: tendencias y controversias. Universidades, 67, 7-24.

Tommasino, H., González Márquez, M. N., Guedes, E., \& Prieto, M. (2006). Extensión crítica: los aportes de Paulo Freire. En Tommasino H. \& Hegedüs, P. (eds.). Extensión: Reflexiones para la intervención en el medio urbano y rural. (pp. 121-135). Montevideo: Universidad de la República. Recuperado de: http://www.documentos.una. ac.cr/bitstream/handle/unadocs/8131/ Extensi\%C3\%B3n-reflexiones\%20para\%20 la $\% 20$ intervenci\%C3\%B3n\%20en $\% 20$ el $\% 20$ medio\%20urbano\%20y\%20rural_Tommasino-De\%20Heged\%C3\%BCs.pdf?sequen$\mathrm{ce}=3 \&$ is Allowed $=\mathrm{y}$

Tommasino, H., Santos, C. \& Stevenazzi, F. (2010). De la extensión a las prácticas integrales. Recuperado de: https://www.researchgate.net/publication/263125629_De_la_extension_a_las_practicas_integrales

Tünnermann Bernheim, C. (2000). El nuevo concepto de la extensión universitaria. Universidad de Michoacana de San Nicolás de Hidalgo Morelia, Michoacán, México., 269. Recuperado de: https://aduba.org. ar/wp-content/uploads/2016/07/NuevoConceptoExtensionUniversitaria-CarlosTunnermann.pdf

Vega Mederos, J. F. (2002). La extensión universitaria: función integradora del quehacer universitario, Investigación \& Desarrollo, 10(1), 26-39. Recuperado de: https://www. redalyc.org/pdf/268/26812103.pdf 
Vera, M. \& Mora, E. (2011). Líneas de investigación en micro, pequeñas y medianas empresas. Revisión documental y desarrollo en Colombia. Tendencias, XII(1), 213-226.

Wenger, E. (2001). Comunidades de práctica. Aprendizaje, significado e identidad. Barcelona: Paidós.

Zevallos, E. (2003). Micro, pequeñas y medianas empresas en América Latina. Revista de la Cepal, 79, 53-70. Recuperado de: http:// repositorio.cepal.org/bitstream/handle/11362/10874/079053070_es.pdf?sequence $=1$

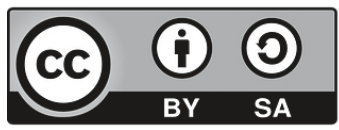

